\title{
Effects of a Self-Monitoring Quality of Life Intervention in Outpatients with Breast Cancer: A Preliminary Report of A Randomized Controlled Trial
}

\author{
Ayako Matsuda ${ }^{1 *}$, Kenichi Inoue ${ }^{2}$, Manami Momiyama $^{2}$, Kunihiko Kobayashi ${ }^{3}$, \\ Kaoru Kubota ${ }^{4}$, Soerindra S.R.S Ramai ${ }^{5}$, Maarten J. Fischer6,7, Judith R. Kroep ${ }^{6}$, \\ Adrian A. Kaptein ${ }^{7}$, Kazue Yamaoka ${ }^{8}$
}

\begin{abstract}
Objective: This preliminary report used data from a randomized controlled clinical trial to investigate the beneficial effects of a self-monitoring quality of life (SMQOL) intervention on communication, medical care and patient outcomes in Japanese women with breast cancer. Methods: This study compared a SMQOL intervention group with a control group that received usual care after 4 months on self-efficacy aspects of patient-physician communication among outpatients with breast cancer in Japan using the Perceived Efficacy in Patient-Physician Interactions (PEPPI) questionnaire. Patients were randomly assigned to the intervention and control groups using permuted-block randomization. The intervention groups were asked to complete a paper-based quality-of-life (QOL) questionnaire in addition to the usual care provided in the control group. Analysis of covariance was used to assess the difference in PEPPI scores between the intervention and control groups. Additionally, subgroup analyses were performed for outpatients with breast cancer accompanied by depression or anxiety. Results: In total, 232 patients were eligible for this study and randomized. Seven patients did not answer the PEPPI questionnaire at baseline after group allocation, leaving 225 patients for inclusion in the analyses. The modified intention-to-treat ITT analysis showed the SMQOL intervention had no significant effect on PEPPI total score $(\mathrm{P}=0.226)$. We found a significant between-group difference in PEPPI total score in the anxiety group $(\mathrm{P}=0.045)$, namely, the self-efficacy aspects of patient-physician communication of those with anxiety in the intervention group were better than for those in the control group after 4 months. Conclusion: Use of the SMQOL had beneficial effects on communication self-efficacy between patients and physicians for outpatients with breast cancer, those with anxiety.
\end{abstract}

Keywords: Physician- patient relationship- self-monitoring- self-efficacy- communication

Asian Pac J Cancer Prev, 23 (1), 53-59

\section{Introduction}

Patient-reported outcomes (PRO), including quality of life (QOL) assessment, are often used as outcome measures in clinical trials, especially for patients with breast cancer. Medical staff may not fully understand the issues and challenges faced by the patients that are reported by PRO. Previous studies suggest that feedback regarding PRO improves patient-physician communication (Rubenstein et al., 1995; Espallargues et al., 2000; Boyes et al., 2006), and routine assessment using measures of PRO in clinical oncology practice has important health benefits for patients (Boyes et al., 2006; Detmar et al., 2002; Gilbody et al., 2002; Velikova et al., 2004; Greenhalgh et al., 2005; Valderas et al., 2008; Fischer et al., 2012). Researches by Temel et al., (2010; 2017) demonstrated important benefits from monitoring QOL in early palliative care of patients with lung cancer and Sullivan et al., (2019) showed that the palliative care was associated with a survival benefit among patients with advanced lung cancer. However, these effects have not been clarified among outpatients with breast cancer.

A care notebook was developed as a way to measure patients' QOL in daily clinical oncology practice

${ }^{1}$ Department of Hygiene and Public Health, Teikyo University School of Medicine, Tokyo, Japan. ${ }^{2}$ Division of Breast Oncology, Saitama Cancer Center, Kitaadachi-gun, Saitama, Japan. ${ }^{3}$ Department of Respiratory Medicine, Saitama Medical University International Medical Center, Hidaka, Saitama, Japan. ${ }^{4}$ Department of Pulmonary Medicine and Oncology Graduate School of Medicine, Nippon Medical School, Tokyo, Japan. ${ }^{5}$ Department of Pulmonology, Leiden University Medical Center, Leiden, the Netherlands. ${ }^{6}$ Department of Medical Oncology, Leiden University Medical Center, Leiden, the Netherlands. ${ }^{7}$ Department of Medical Psychology, Leiden University Medical Center, Leiden, the Netherlands. ${ }^{8}$ Teikyo University Graduate School of Public Health,Tokyo, Japan.*For Correspondence: amatsuda@med.teikyo-u.ac.jp 
(Kobayashi et al., 2005). The Care Notebook allows clinical oncologists to easily collect valid and reliable QOL information with a minimal burden on patients (Kobayashi et al., 2005). The Department of Medical Oncology of Leiden University Medical Centre in the Netherlands has implemented a self-monitored QOL (SMQOL) intervention using the Care Notebook.

In our study, we used data from a randomized controlled trial (RCT) that aimed to investigate the effects of SMQOL in early breast cancer in Netherlands and Japan. This RCT was an international collaborative study between Netherlands and Japan, which aimed to investigate the beneficial effects of the SMQOL intervention on aspects of communication, medical care, and outcomes in patients with early breast cancer in the Netherlands and Japan who were treated with adjuvant or neo-adjuvant chemotherapy (Lugtenberg et al., 2020). Preliminary results on the assessment of the extent to which QOL-topics in the primary objective of this study have been published for Dutch patients treated in the Netherlands (Lugtenberg et al., 2020). In our article, we report the results for Japanese patients as a preliminary analysis in the effectiveness of the SMQOL intervention on self-efficacy of communication between physicians and patients, specifically outpatients with breast cancer. The primary objective of the collaborative study was to assess the extent to which QOL-topics and secondary objectives were the assessment of outcomes using the Perceived Efficacy in Patient-Physician Interactions (PEPPI) and the Hospital Anxiety and Depression Scale (HADS) questionnaires. The present study was one of the secondary objectives of the collaborative study; however, we were unable to assess the primary objective owing to the lack of a considerable amount of data.

A previous study investigating a SMQOL intervention for patients with cancer receiving palliative did not show a significant overall effect on global QOL in an intention to treat (ITT) analysis (Matsuda et al., 2019). When evaluating the effects of a SMQOL intervention, it is important to consider the self-efficacy aspects of communication between patients and physicians as a PRO. For instance, Detmar et al., (2002) described how QOL assessments in daily clinical oncology practice facilitate discussion of QOL issues, and can heighten physicians' awareness of their patients' QOL in patient-physician communication. An important feature of our study is that we assessed the self-efficacy of communication between patients and physicians using a SMQOL intervention. Previous studies have reported that women are most vulnerable to psychological morbidity at diagnosis (Stafford et al., 2013). Intensive psychological intervention is necessary for patients with breast cancer as well as for women with suspected breast cancer with high scores on HADS at preconsultation (Iwatani et al., 2013). Therefore, we also focused on the effectiveness of the SMQOL intervention for outpatients with breast cancer that also had depression or anxiety (as indicated by HADS scores).

The present study aimed to evaluate the effectiveness of SMQOL intervention after 4 months on self-efficacy of communication between patients and physicians among outpatients with breast cancer in Japan. Additionally, we conducted subgroup analyses for participants with depression or anxiety.

\section{Materials and Methods}

\section{Study design}

Details of the study design have been previously published (Lugtenberg et al., 2020). The trial design followed the Standard Protocol Items: Recommendations for Interventional Trials (SPIRIT) 2013 statement (Chan et al., 2013). The prospective RCT started at Saitama Cancer Center in July 2014 and concluded in November 2016. All participants were asked to provide written informed consent to participate in the study. After providing consent and completing a case report form (CRF) with the researchers, patients were randomized to an intervention group (SMQOL) or a control group (usual care).

The CRF covered items such as age, Eastern Cooperative Oncology Group performance status (PS) cancer stage (I, II, or III); estrogen receptor (ER) and/ or progesterone receptor (PR) (positive or no); human epidermal growth factor receptor 2 (HER2) (positive or no); timing for the implementation of chemotherapy (adjuvant or neo-adjuvant); and previous radiotherapy (yes or no).

\section{Study setting}

After obtaining ethical approval from the Institutional Review Board of Saitama Cancer Center (CA014-14), the trial was registered with the UMIN clinical trials registry (trial registration number: UMIN000026212. Issue Date: February 19, 2017).

\section{Patients and eligibility criteria}

Eligibility criteria included patients aged 20 years, scheduled to receive neo-adjuvant or adjuvant chemotherapy or over who were in a physical condition that allowed them to tolerate the investigation.

Randomization and blinding

Eligible participants were randomly assigned using a permuted-block technique with a randomization list (random permutated blocks with a block size of four) (Hully et al., 2001). Allocation to the intervention group was performed by the principal researcher. Eligible patients and researchers were not informed as to which group patients were randomized, but no blinding was performed.

\section{Intervention \\ Intervention group}

Patients randomly assigned to the intervention group were asked to complete a paper-based QOL-monitor in addition to usual care. Patients completed the QOL-monitor before every consecutive visit (starting at the end of the second cycle). The QOL-monitor included the following items: the Care Notebook (Care Notebook Center: http:// www.care-notebook.com/en/download.html) (Kobayashi et al., 2005), the European Organization for Research and Treatment of Cancer Breast Cancer-specific QOL questionnaire (BR-23) (Sprangers et al., 1996), the 
National Comprehensive Cancer Network Distress Thermometer (Roth et al., 1998), one free text dialog box, and one question assessing additional supportive care needs. Detailed information on this intervention has been previously published (Lugtenberg et al., 2020).

\section{Control group}

The control group received "usual care" or routine practice that medical staff provides during course of routine clinical care for patients with breast cancer.

\section{Outcome measures}

We used the PEPPI and the HADS questionnaires as the outcome measures. These scales were part of the questionnaires that were used in the RCT.

\section{PEPPI}

Self-efficacy for communication between patients and physician was reported by patients, and assessed using the PEPPI questionnaire (Maly et al., 1998; Matsuda et al., 2019). Participants responded to each question on a scale from 1 to 5, with 5 representing "very confident," and 1 representing "not at all confident." The range of possible scores for the full PEPPI scale is 10-50, with 50 representing the highest level of patient-perceived self-efficacy.

\section{HADS}

Anxiety and depression were measured by the HADS (Zigmond and Snaith, 1983; Kugaya et al., 1998). The HADS performs well in assessing symptom severity and presence of anxiety disorders and depression in somatic, psychiatric, and primary care patients, as well as in the general population. The HADS is a four-point, 14-item self-assessment scale to measure psychological distress, and has two factors. The instrument is divided into anxiety and depression subscales, each containing seven intermingled items. Each item scores on a 4-point Likert scale, giving maximum subscale scores of 21 for depression and anxiety, respectively (Zigmond and Snaith, 1983; Kugaya et al., 1998). The cut-off point of the anxiety subscale is $7 / 8$ and the cut-off point of the depression subscale is 10/11 (Zigmond and Snaith, 1983).

\section{Data collection and time points}

Sociodemographic data for eligible participants were collected at baseline (after group allocation). The outcomes were also collected at baseline (after group allocation), and at 2 and 4 months for both the control and intervention groups. The outcomes reported in this study were assessed at baseline and 4 months in both the control and intervention groups.

\section{Participant characteristics}

Sociodemographic data included age, marital status, and employment at time of diagnosis. Clinical data were obtained from the CRF.

\section{Statistical analysis}

In terms of the sample size, as our study used data from our previous RCT, a formal power analysis was not performed. The sample size used in our previous RCT was considered a feasible sample size.

The outcomes were analyzed following a modified ITT principle. For the modified ITT (Dossing et al., 2006), we excluded patients who did not complete the PEPPI questionnaire at baseline (after group allocation). The last observation carried forward (LOCF) method was used to manage missing data due to attrition (Lydersen S., 2019). Specifically, if an observation was missing, the last observed value was imputed at future time points where it was missing.

Analysis of covariance (ANCOVA) was used to assess the difference in PEPPI scores between the intervention and control groups. The PEPPI total score at 4 months was used as the dependent variable, and the baseline PEPPI score and group assignment were used as fixed factors for the independent variables in a simple model. In addition to the simple model, we used a full model adjusted for age, cancer stage, ER and/or PR, HER2, timing of chemotherapy, previous radiotherapy, marital status, and employment at the time of diagnosis as covariates. Additionally, we conducted ANCOVA with simple and full models for sub-analyses with anxiety/ no anxiety groups and depression/no depression groups. Those with a HADS-Anxiety score of $<8$ were classified as the no anxiety group, whereas a HADS-Anxiety score of $\geq 8$ was classified as the anxiety group. Those with a HADS-Depression score of $<11$ were classified as the no depression group, whereas a HADS-Depression score $\geq 11$ was classified as the depression group (Zigmond and Snaith, 1983). $\mathrm{P}<0.05$ (two-sided) was considered statistically significant. Statistical analyses were performed with SAS version 9.4 (SAS Institute Inc., Cary, NC, USA).

\section{Results}

\section{Study participation}

Patients were enrolled from October 2014 to November 2016. A flow-chart of randomized participating patients is presented in Figure. 1. In total, 232 patients were considered eligible and randomized; 116 each in the control and intervention groups. Seven patients (control group: $\mathrm{n}=4$, intervention group $\mathrm{n}=3$ ) did not answer the PEPPI questionnaires at baseline after group allocation, leaving 225 patients for inclusion in the analysis (control group: $\mathrm{n}=112$, intervention group: $\mathrm{n}=113$ ).

\section{Patient characteristics}

The characteristics of participating patients at baseline are shown in Table 1. The mean age in the intervention group was 52.0 years and that in the control group was 54.3 years. The majority of patients had cancer stage I or II, were hormone receptor positive and HER2 negative, receiving adjuvant chemotherapy, and had not previously received radiotherapy. Most patients were married or had a partner, and about half were employed at the time of diagnosis. The mean (standard deviation) PEPPI total score, HADS-Anxiety score, and HADS-Depression score at baseline for patients in the intervention and control groups were: 34.4 (7.1) and 34.6 (7.1), 6.9 (3.6) and 7.7 
Table 1. Baseline Characteristics in the Modified Intention to Treat Population $(\mathrm{N}=225)$

\begin{tabular}{|c|c|c|c|c|c|}
\hline & & \multicolumn{2}{|c|}{ Intervention group $(\mathrm{n}=113)$} & \multicolumn{2}{|c|}{ Control group $(\mathrm{n}=112)$} \\
\hline \multicolumn{6}{|l|}{ Sociodemographic data } \\
\hline Age, mean (SD) & & 52 & -10.1 & 54.3 & -10.3 \\
\hline Partnered/married, n (\%) & & 102 & -90.3 & 104 & -92.9 \\
\hline Employed at time of diagnosis, $\mathrm{n}(\%)$ & & 64 & -56.6 & 65 & -58 \\
\hline \multicolumn{6}{|l|}{ Clinical data } \\
\hline \multicolumn{6}{|c|}{ Eastern Co-operative Oncology Group performance status, n (\%) } \\
\hline & 0 & 113 & -100 & 112 & -100 \\
\hline \multicolumn{6}{|l|}{ Cancer stage, $\mathrm{n}(\%)$} \\
\hline & I & 26 & -23 & 21 & -18.8 \\
\hline & II & 72 & -63.7 & 72 & -64.3 \\
\hline & III & 15 & -13.3 & 19 & -17 \\
\hline ER* and/or PR**, positive, $\mathrm{n}(\%)$ & & 81 & -71.7 & 69 & -61.6 \\
\hline HER2 $* * *$ positive, $\mathrm{n}(\%)$ & & 34 & -30.1 & 42 & -37.5 \\
\hline \multicolumn{6}{|c|}{ Timing for the implementation of chemotherapy, $\mathrm{n}(\%)$} \\
\hline & Adjuvant & 81 & -71.7 & 80 & -71.4 \\
\hline & Neo-adjuvant & 32 & -28.3 & 32 & -28.6 \\
\hline \multirow[t]{2}{*}{ Previous radiotherapy, $\mathrm{n}(\%)$} & & 12 & -10.6 & 15 & -13.4 \\
\hline & Missing value & 2 & -1.8 & 2 & -1.8 \\
\hline PEPPI $\dagger$ & & 34.4 & -7.1 & 34.6 & -7.1 \\
\hline HADS $\$$-Anxiety, mean (SD) & & 6.9 & -3.6 & 7.7 & -4 \\
\hline HADS:-Depression, mean (SD) & & 9.4 & -3.4 & 10 & -3.8 \\
\hline
\end{tabular}

SD, standard deviation; Abbreviations: *ER, estrogen receptor; **PR, progesterone receptor; ***HER2, human epidermal growth factor receptor 2; †PEPPI, Perceived Efficacy in Patient-Physician Interactions; $\$$ HADS, Hospital Anxiety and Depression Scale

(4.0), and $9.4(3.4)$ and $10.0(3.8)$, respectively.

Modified ITT and subgroup analyses

In the simple model, the modified ITT, anxiety/no anxiety, and depression/no depression groups showed the SMQOL intervention had no significant effect on PEPPI total score. In the full model, we additionally adjusted for age, cancer stage, ER/PR, HER2, timing of chemotherapy,

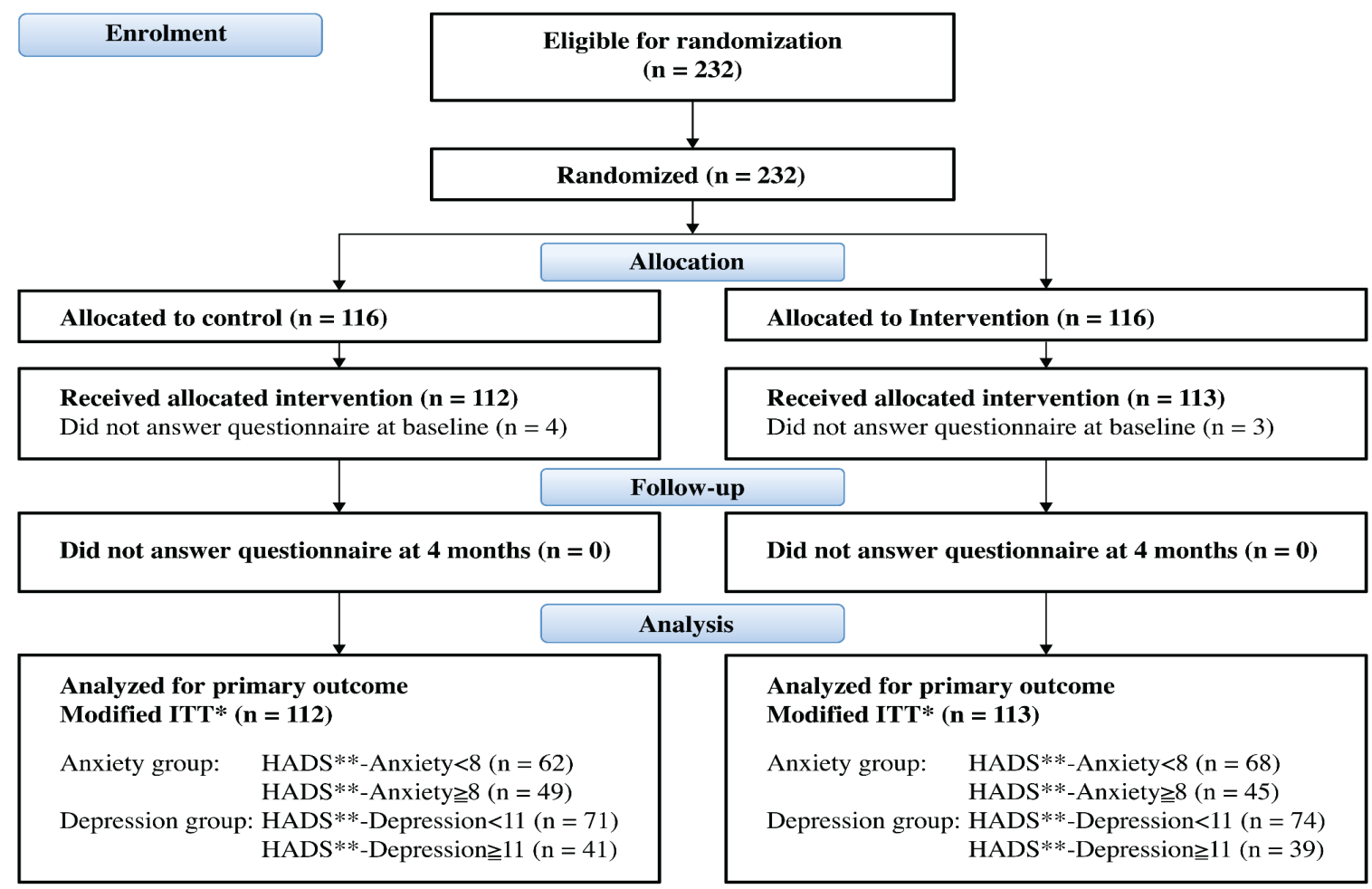

Figure 1. The study flow. * ITT, intention to treat; **HADS, Hospital Anxiety and Depression Scale 



$($ HADS $†$-Depression $\geqq 11)$

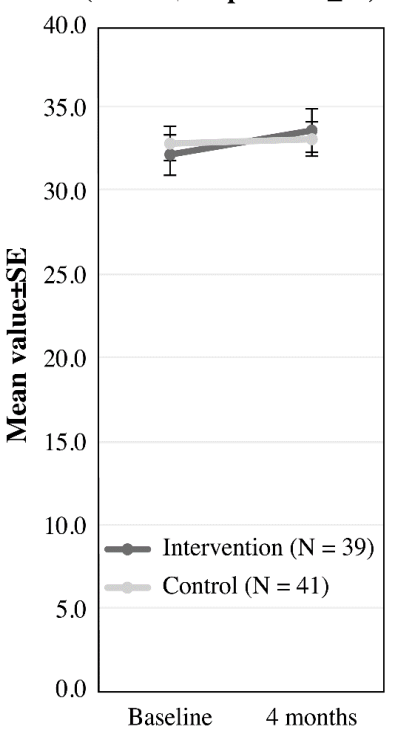

Figure. 2 Changes in Perceived Efficacy in Patient-Physician Interactions (PEPPI) scores of patients over time: mean values of individual changes. Abbreviations: SE, standard error; $\uparrow$ HADS, Hospital Anxiety and Depression Scale

previous radiotherapy, marital status, and employment at the time of diagnosis. This model revealed a significant between-group difference in PEPPI total score for the anxiety group (difference between the groups: 2.36 , $\mathrm{P}=0.045$ ) (Table 2)

Among patients with anxiety, the PEPPI total score for those in the intervention group was higher than the control group at 4 months. The modified ITT analysis showed no significant differences between the intervention and control groups in the no anxiety, no depression, and depression groups. The results are presented in Table 2 and Figure 2.

\section{Discussion}

In the group of outpatients with breast cancer that also had anxiety, routine use of SMQOL improved self-efficacy for communication between patients and physicians. However, in the group with depression, the SMQOL intervention did not significantly improve communication self-efficacy, although the PEPPI total score in the intervention group tended to be higher than that in the control group at 4 months (Figure 2). Our finding supported our hypothesis that the SMQOL intervention improved the self-efficacy for patientphysician communication. Our findings will help to address the gap in research on SMQOL interventions for outpatients with breast cancer, especially those that also experience depression or anxiety. Increased knowledge regarding SMQOL for this patient population will support improved patient-physician communication. A previous study reported that if clinicians rely on patients to initiate discussion of psychosocial issues, patients' problems may go unaddressed (Taylor et al., 2011). Our results

Table 2. Estimates of the Intervention Effects on Perceived Efficacy in Patient-Physician Interactions Scores on Self-Monitoring Quality of Life Intervention after 4 Months

\begin{tabular}{|c|c|c|c|c|}
\hline Simple model & & Difference between the groups & $95 \% \mathrm{CI}$ & P-value $\dagger$ \\
\hline Modified ITT $\S$ & $(n=225)$ & 0.64 & -0.77 to 2.05 & 0.372 \\
\hline No anxiety group & HADS"-Anxiety < $8(\mathrm{n}=130)$ & -0.08 & -1.95 to 1.78 & 0.931 \\
\hline Anxiety group & HADS"-Anxiety $\geq 8(\mathrm{n}=94)$ & 1.78 & -0.33 to 3.89 & 0.098 \\
\hline No depression group & HADS"-Depression < $11(\mathrm{n}=145)$ & 0.31 & -1.4 to 2.02 & 0.721 \\
\hline Depression group & HADS"-Depression $\geq 11(\mathrm{n}=80)$ & 1.16 & -1.35 to 3.66 & 0.36 \\
\hline Full model & & Difference between the groups & $95 \% \mathrm{CI}$ & P-valuet: \\
\hline Modified ITT $\$$ & $(\mathrm{n}=222)$ & 0.89 & -0.56 to 2.35 & 0.226 \\
\hline No anxiety group & HADS"-Anxiety $<8(\mathrm{n}=128)$ & 0.02 & -1.92 to 1.96 & 0.982 \\
\hline Anxiety group & HADS"-Anxiety $\geq 8(\mathrm{n}=93)$ & 2.36 & 0.06 to 4.66 & 0.045 \\
\hline No depression group & HADS"-Depression $<11(\mathrm{n}=145)$ & 0.36 & -1.43 to 2.15 & 0.693 \\
\hline Depression group & HADS"-Depression $\geq 11(\mathrm{n}=77)$ & 1.74 & -0.91 to 4.40 & 0.194 \\
\hline
\end{tabular}

Abbreviations: CI: confidence interval; "Analysis of covariance was used; $\uparrow$ P value: Simple model: adjusted for baseline of PEPPI; $\$$ P-value: Full model: simple model + age, cancer stage (I, II, III), estrogen receptor and/or progesterone receptor (positive or no), human epidermal growth factor receptor 2 (positive or no), timing of chemotherapy (adjuvant or neo-adjuvant), previous radiotherapy (Yes or No), marital status (Yes or No), and employed at time of diagnosis (Yes or No)."§ ITT, intention to treat; ${ }^{\uparrow}$ HADS, Hospital Anxiety and Depression Scale 
suggested that SMQOL may benefit patient-physician communication, and use of SMQOL was effective and practical in outpatients with breast cancer with depression or anxiety. A previous study showed that routine assessment of health-related QOL among patients with cancer had an impact on physician-patient communication (Velikova et al., 2004; Epstein et al., 2017). Routine use of SMQOL in clinical practice may also facilitate improvements in patient-physician communication as a communication tool.

Patients with breast cancer undergoing hormone therapy might experience serious psychological symptoms (Iioka et al., 2020). Preliminary results of the RCT indicated that the use of SMQOL during breast cancer treatment with chemotherapy might lead to more frequent discussions of health-related QOL topics, especially regarding psychosocial and breast cancer-specific issues (Lugtenberg et al., 2020). An intervention such as SMQOL may be needed in clinical practice for patients with breast cancer that also have depression or anxiety. The present study provided evidence for the use of SMQOL in clinical practice for these patients. The previous study described that a preconsultation educational group intervention improved patients' shared decision-making quality (Causarano et al., 2015). The use of SMQOL in clinical practice might be useful for decision-making during treatment.

Finally, there were some limitations in this study. The present study did not use a double-blind design, but eligible patients and researchers were not informed to which group patients were randomized until the study started. Additionally, the outcome measures were clearly set and used reliable scales.

The collaborative study was powered for the total group of Japanese and Dutch patients and not for this subgroup, nor for all the secondary outcome measures. The present study was assessed as PEPPI of the secondary outcome measure. A sample size of 225 per group at 4 months follow-up was calculated to provide $80 \%$ power to detect statistically significant differences in mean levels of primary outcomes between the two groups (as small as 2.5 of the applicable standard deviation, using two-sided 0.05 significance tests). These power calculations were based on a simple two-group comparison of a single outcome variable at the 4-month follow-up visit with an independent t-test. However, the sample size did not have sufficient power because the estimated standard deviation of PEPPI total score was 11.1, and the minimum detectable difference was 1.0. The modified ITT analysis showed no significant effect on PEPPI total score, because the estimated standard deviation was larger than the expected value. However, the information about the effect size of PEPPI in our study will probably be useful for any plan to calculate sample size. Additionally, a previous study showed that a modified ITT analysis allowed a systematically less biased approach to evaluate the effects of an intervention (Currow et al., 2012).

Our study suggests the SMQOL has practical use for outpatients with breast cancer that have depression or anxiety. Worldwide, there are limited SMQOL measures available for outpatients with cancer. The SMQOL intervention that was used in our study may help to address this lack. Our findings help to clarify the practical use of a SMQOL intervention for outpatients with breast cancer that also have depression or anxiety. We recommend the use of a SMQOL intervention for outpatients with breast cancer that have depression or anxiety in healthcare facilities.

\section{Acknowledgements}

The present study was supported by the Ministry of Education, Culture, Sports, Science and Technology in Japan Grant-in-Aid for Scientific Research Grant B in 2015 (grant number: 15K19295).

The authors have no financial relationship with the organizations that sponsored this research.

\section{Author Contribution Statement}

AM conceived the study. All authors participated in the development of the protocol. KI and MM collected and cleaned the data, and obtained ethics approval and consent. AM and KY analyzed the data. AM and KY wrote the first draft of the paper. KK, KK, and KI edited the manuscript and provided expert advice based on their medical specialist knowledge. All authors prepared and revised the manuscript, including relevant scientific content. All authors approved the final version of the manuscript.

\section{Funding Statement}

The present study was supported by the Ministry of Education, Culture, Sports, Science, and Technology in Japan Grant-in-Aid for Scientific Research Grant B in 2015 (grant number: 15K19295).

\section{Ethics Approval and Consent to Participate}

This study was conducted in accordance with the Declaration of Helsinki and was approved by the Ethics Committee of all participating institutes in agreement for medical research involving human subjects. All patients signed informed consent before any study related procedures.

\section{Consent for Publication \\ Not applicable}

\section{Availability of Data and Materials}

The datasets used and/or analyzed during the current study are available from the corresponding author on reasonable request.

\section{References}

Boyes A, Newell S, Girgis A, McElduff P, Sanson-Fisher R (2006). Does routine assessment and real-time feedback improve cancer patients' psychosocial well-being? Eur J Cancer Care, 15, 163-71.

Causarano N, Platt J, Baxter NN, et al (2015). Pre-consultation educational group intervention to improve shared decisionmaking for postmastectomy breast reconstruction: a pilot 
randomized controlled trial. Support Care Cancer, 23, 1365-75.

Chan AW, Tetzlaff JM, Altman DG, et al (2013). Spirit 2013 statement: defining standard protocol items for clinical trials. Ann Intern Med, 158, 200-7.

Currow DC, Plummer JL, Kutner JS, Samsa GP, Abernethy AP (2012). Analyzing phase iii studies in hospice/palliative care. A solution that sits between intention-to-treat and per protocol analyses: the palliative-modified itt analysis. J Pain Symptom Manage, 44, 595-603.

Detmar SB, Muller MJ, Schornagel JH, Wever LD, Aaronson NK (2002). Health-related quality-of-life assessments and patient-physician communication: a randomized controlled trial. JAMA, 288, 3027-34.

Dossing A, Tarp S, Furst DE, et al (2016). Modified intentionto-treat analysis did not bias trial results. J Clin Epidemiol, 72, 66-74.

Epstein RM, Duberstein PR, Fenton JJ, et al (2017). Effect of a patient-centered communication intervention on oncologistpatient communication, quality of life, and health care utilization in advanced cancer: the voice randomized clinical trial. JAMA Oncol, 3, 92-100.

Espallargues M, Valderas JM, Alonso J (2000). Provision of feedback on perceived health status to health care professionals: a systematic review of its impact. Med Care, 38, $175-86$.

Fischer MJ, Krol-Warmerdam EM, Ranke GM, et al (2012). Routine monitoring of quality of life for patients with breast cancer, an acceptability and field test. J Psychosoc Oncol, 30, 239-59.

Gilbody SM, House AO, Sheldon T (2002). Routine administration of health-related quality of life (hrqol) and needs assessment instruments to improve psychological outcome--a systematic review. Psychol Med, 32, 1345-56.

Greenhalgh J, Long AF, Flynn R (2005). The use of patient reported outcome measures in routine clinical practice: lack of impact or lack of theory?. Soc Sci Med, 60, 833-43.

Hully SB, Cummings SR, Browner WS, Grady DG, Newman T B (2001). Designing Clinical Research: An Epidemiologic Approach, 2nd Ed. In 'Cummings', Eds Gradty D and Hully SH. Lippincott Williams \& Wilkins, Philadelphia, pp 155-8.

Iioka Y, Iwata T, Yamauchi H (2020). Symptoms and qol in breast cancer patients receiving hormone therapy in japan. Breast Cancer, 27, 62-9.

Iwatani T, Matsuda A, Kawabata H, Miura D, Matsushima E (2013). Predictive factors for psychological distress related to diagnosis of breast cancer. Psychooncology, 22, 523-9.

Kobayashi K, Green J, Shimonagayoshi M, et al (2005). Validation of the care notebook for measuring physical, mental and life well-being of patients with cancer. Qual Life Res, 14, 1035-43.

Kugaya A, Akechi T, Okuyama T, Okamura H, Uchitomi Y (1998). Screening for psychological distress in japanese cancer patients. Jpn J Clin Oncol, 28, 333-8.

Lugtenberg RT, Fischer MJ, de Jongh F, et al (2020). Using a quality of life (qol)-monitor: preliminary results of a randomized trial in dutch patients with early breast cancer. Qual Life Res, 29, 2961-75.

Lydersen S (2019). Last observation carried forward. Tidsskr Nor Laegeforen, 139.

Maly RC, Frank JC, Marshall GN, DiMatteo MR, Reuben DB (1998). Perceived efficacy in patient-physician interactions (peppi): validation of an instrument in older persons. $J \mathrm{Am}$ Geriatr Soc, 46, 889-94.

Matsuda A, Inoue K, Momiyama M, et al (2019). Validity and reliability of the japanese version of the 10-item perceived efficacy in patient-physician interactions (peppi-10) scale in breast cancer outpatients. Tohoku J Exp Med, 249, 121-6.

Matsuda A, Yamada Y, Ishizuka N, et al (2019). Effectiveness of a self-monitoring quality of life intervention for patients with cancer receiving palliative care: a randomized controlled clinical trial. Asian Pac J Cancer Prev, 20, 2795-802.

Roth AJ, Kornblith AB, Batel-Copel L, et al (1998). Rapid screening for psychologic distress in men with prostate carcinoma: a pilot study. Cancer, 82, 1904-8.

Rubenstein LV, McCoy JM, Cope DW, et al (1995). Improving patient quality of life with feedback to physicians about functional status. $J$ Gen Intern Med, 10, 607-14.

Sprangers MA, Groenvold M, Arraras JI, et al (1996). The european organization for research and treatment of cancer breast cancer-specific quality-of-life questionnaire module: first results from a three-country field study. J Clin Oncol, 14, 2756-68.

Stafford L, Judd F, Gibson P, et al (2013). Screening for depression and anxiety in women with breast and gynaecologic cancer: course and prevalence of morbidity over 12 months. Psychooncology, 22, 2071-78.

Sullivan DR, Chan B, Lapidus JA, et al (2019). Association of early palliative care use with survival and place of death among patients with advanced lung cancer receiving care in the veterans health administration. JAMA Oncol, 5, 1702-09.

Taylor S, Harley C, Campbell LJ, et al (2011). Discussion of emotional and social impact of cancer during outpatient oncology consultations. Psychooncology, 20, 242-51.

Temel JS, Greer JA, Muzikansky A, et al (2010). Early palliative care for patients with metastatic non-small-cell lung cancer. N Engl J Med, 363, 733-42.

Temel JS, Greer JA, El-Jawahri A, et al (2017). Effects of early integrated palliative care in patients with lung and gi cancer: a randomized clinical trial. J Clin Oncol, 35, 834-41.

Valderas JM, Kotzeva A, Espallargues M, et al (2008). The impact of measuring patient-reported outcomes in clinical practice: a systematic review of the literature. Qual Life Res, 17, 179-93.

Velikova G, Booth L, Smith AB, et al (2004). Measuring quality of life in routine oncology practice improves communication and patient well-being, a randomized controlled trial. J Clin Oncol, 22, 714-24.

Zigmond AS, Snaith RP (1983). The hospital anxiety and depression scale. Acta Psychiatr Scand, 67, 361-70.

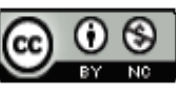

This work is licensed under a Creative Commons AttributionNon Commercial 4.0 International License. 